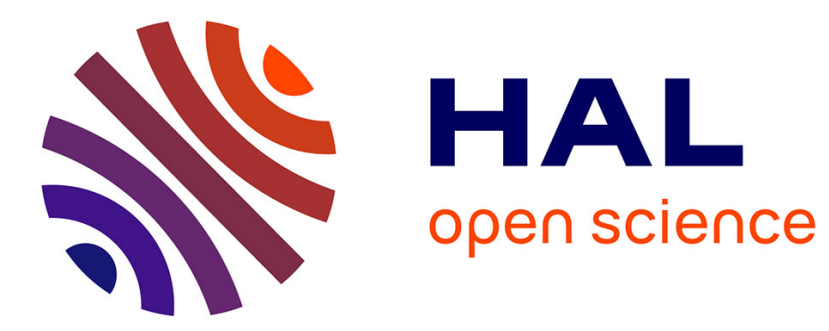

\title{
Force distribution on multiple bonds controls the kinetics of adhesion in stretched cells.
}

Daniel Isabey, Sophie Féréol, Adam Caluch, Redouane Fodil, Bruno Louis, Gabriel Pelle

\section{- To cite this version:}

Daniel Isabey, Sophie Féréol, Adam Caluch, Redouane Fodil, Bruno Louis, et al.. Force distribution on multiple bonds controls the kinetics of adhesion in stretched cells.. Journal of Biomechanics, 2013, 46 (2), pp.307-13. 10.1016/j.jbiomech.2012.10.039 . inserm-00766438

\section{HAL Id: inserm-00766438 https://www.hal.inserm.fr/inserm-00766438}

Submitted on 18 Dec 2012

HAL is a multi-disciplinary open access archive for the deposit and dissemination of scientific research documents, whether they are published or not. The documents may come from teaching and research institutions in France or abroad, or from public or private research centers.
L'archive ouverte pluridisciplinaire HAL, est destinée au dépôt et à la diffusion de documents scientifiques de niveau recherche, publiés ou non, émanant des établissements d'enseignement et de recherche français ou étrangers, des laboratoires publics ou privés. 
This article is for the special issue on Biofluid Mechanics

\section{Force distribution on multiple bonds controls the kinetics of adhesion in stretched cells}

Isabey $D^{\alpha^{*}}$, Féréol $S^{\&}$, Caluch $A^{\&}$, Fodil $R^{\&}$, Louis $B^{\&}$, Pelle $G^{\text {\&* }}$

\& Inserm UMR955, Mondor Institute of Biomedical Research, Cell and Respiratory Biomechanics Laboratory, Créteil, 94010, France

\& CNRS URL 7240, Cell and Respiratory Biomechanics Laboratory, Créteil, 94010, France

\& Université Paris Est Créteil, Faculté de Médecine, Créteil, 94010, France

* AP-HP, Hôpital Henri Mondor, Service de Physiologie - Explorations Fonctionnelles, Créteil, 94010 France

${ }^{*}$ Correspondence address:

Daniel Isabey

Inserm UMR 955,

Equipe Biomécanique Cellulaire et Respiratoire

Faculté de Médecine

8, rue du Général Sarrail

94010 CRETEIL Cedex, France

Phone: 33149813700

Fax: 33148981777

E-mail: daniel.isabey@inserm.fr

Key words: parallel bonds, zipper bonds, lifetime, dissociation rate, bond strength. 


\section{Abstract}

We show herein how mechanical forces at macro or micro scales may affect the biological response at the nanoscale. The reason resides in the intimate link between chemistry and mechanics at the molecular level. These interactions occur under dynamic conditions such as the shear stress induced by flowing blood or the intracellular tension. Thus, resisting removal by mechanical forces, e.g., shear stresses, is a general property of cells provided by cellular adhesion. Using classical models issued from theoretical physics, we review the force regulation phenomena of the single bond. However, to understand the force regulation of cellular adhesion sites, we need to consider the collective behavior of receptor-ligand bonds. We discuss the applicability of single bond theories to describe collective bond behavior. Depending on bond configuration, e.g., presently "parallel" and "zipper", the number of bonds and dissociation forces variably affect the kinetics of multiple bonds. We reveal a marked efficiency of the collective organization to stabilize multiple bonds by sharply increasing bond lifetime compared to single bond. These theoretical predictions are then compared to experimental results of the literature concerning the kinetic parameters of bonds measured by atomic force microscopy and by shear flow. These comparisons reveal that the force-control of bonds strongly depends on whether the force distribution on multiple bonds is homogeneous, e.g., in AFM experiments, or heterogeneous, e.g., in shear flow experiments. This reinforces the need of calculating the stress/strain fields exerted on living tissues or cells at various scales and certainly down to the molecular scale. 


\section{Introduction}

It is widely accepted that mechanical forces control normal biological processes and their pathological alterations through cell-environment interactions (Discher et al., 2005). These interactions occur under dynamic conditions, e.g., the flowing blood; the internal tension, through adhesion sites which are protein assemblies through clusters which link the cytoskeleton to the extracellular environment (Geiger and Bershadsky, 2001). In particular, receptor-ligand bond anchoring cells in the presence of flowing liquids are stretched by tensile mechanical forces that balance the drag force on the bound cell. Therefore, stabilization under force and resisting removal to shear stresses - and to intra/extracellular stretching forces - are fundamental properties of cellular adhesion. Moreover, weakening or strengthening adhesion by mechanical force remains an opened question if one wants to understand flow-controlled key cellular interactions such as: macrophage-epithelium, leukocyte-endothelium or bacteria-host cell (Thomas, 2008; Thomas et al., 2008).

A fundamental aspect to consider is the stochastic nature of molecular adhesion and the subsequent bond detachment under force which are extensively described in the literature (Bongrand and Golstein, 1983; Evans, 1998). A less considered aspect deals with the complexity of adhesion structures since cellular adhesion involves not only one but several bonds, which stabilize and resist mechanical forces through different strategies of association and reinforcement (Evans, 2001; Evans and Ritchie, 1994; Leckband et al., 1992). These strategies are most likely influenced by force transiting through intra/extracellular environment and a number of biochemical and biomechanical processes implicating signaling pathways (Vogel and Sheetz, 2009). From initial adhesion to focal complex and beyond the mature focal adhesion, the adhesion site configuration changes and the maximal 
force supported by adhesion sites varies by six orders of magnitude ( $\mathrm{pN}-\mu \mathrm{N})$ (Bruinsma, 2005). Although these adhesion sites are made of similar weak bond units - i.e., a non covalent molecular link given to support forces up to the $\mathrm{pN}$ - their collective organization allows them supporting a huge range of stress (Geiger and Bershadsky, 2001). The structural changes associated to stabilization and strengthening include: (i) increase in cell-substrate contact area (spreading) (Capo et al., 1981), (ii) recruitment of receptors to anchoring sites including their lateral association (clustering) (Andre et al., 1990; Bell et al., 1984; Cluzel et al., 2005), (iii) interaction with cytoskeleton elements that lead to enhanced force distribution among bound receptors via local cytoskeleton stiffening (focal adhesion assembly) (Pasternak and Elson, 1985). These mechanisms of adhesion strengthening are supported by numerous observations from various cellular systems (Balaban et al., 2001; Choquet et al., 1997; Galbraith et al., 2002; Giannone et al., 2003; Zhu et al., 2008). Trying to understand these mechanisms of adhesion stabilization or reinforcement, and how they are regulated by force, remains challenging questions which require new theoretical knowledge and pertinent analysis of data.

Recognizing the need to expose actions at the submicroscopic level, many research groups have employed ultrasensitive force techniques to probe extremely small regions of adhesive contact between surfaces functionalized by biological molecules (Evans and Ritchie, 1994; Leckband et al., 1992). Most direct measurements of single bond strength have been performed with three types of ultrasensitive probes: the Atomic Force Microscope (AFM) where force is sensed by deflection of a thin silicon nitride cantilever (Binnig et al., 1986), the Biomembrane Force Probe (BFP) where force is sensed by a glass microsphere glued to the pole of a micropipette-pressurized membrane capsule (Evans et al., 1995; Simson et al., 1998); and the laser optical tweezers where force is sensed by displacement of a 
microsphere trapped in a narrowly focused beam of laser light (Ashkin, 1992; Ashkin et al., 1990). Common limitations associated with these techniques deal with thermal fluctuations, hydrodynamic interactions, and noteworthy the difficulty of controlling the number and the configuration of bonds implicated.

Since most of adhesive interactions are initiated and then sustained under flow conditions (in priority blood but no only), cell resistance to removing by fluid shear stress using a "laminar flow chamber" has become a relevant method to measure the kinetics of receptor-ligand adhesion (Bongrand and Golstein, 1983; Pierres et al., 1996a; Pierres et al., 1996b). Basically, particles or circulating cells covered by receptors are flowing near a surface coated by a ligand and arrested by the formation of limited number of adhesion bonds, e.g., one per cell in many experiments, as far as hydrodynamic forces do not exceed a few pNs (Kaplanski et al., 1993; Pierres et al., 1996a; Smith et al., 1999). The relationship between the number of arrested particles and the duration of their arrest allows (i) determining detachment curves whose initial slope provides the dissociation rate (Chang and Hammer, 1999; Smith et al., 1999; Tissot et al., 1992) and moreover the (ii) study the mechanisms of flow-enhanced cell adhesion (Zhu et al., 2008).

The present paper concerns some under considered aspects of the regulation by mechanical force of collective bond organization implicated in the structuration of different adhesion sites. We purposely apply theories of bond dissociation - issued from stochastic approaches - to different types of bond configurations, e.g., parallel or zipper bonds, on which dissociation forces are exerted. The experimental data purposely used are issued from two different methods: the atomic force microscopy (AFM) and the laminar (viscous) shear flow. It appears that force-control of multiple bonds strongly depends on whether the force 
distribution on multiple bonds is homogeneous, such as during certain AFM experiments, or heterogeneous, such as during shear flow experiments. Thus, to understand the cell adhesion response to stretch, the knowledge of force distribution is needed down to the smallest scale possible. This reinforces the need of calculating the stress/strain fields exerted on living tissues or cells at various scales and certainly down to the molecular scale.

\section{Methods}

\section{Kinetic theory of bond dissociation}

\section{Single bond}

Weak noncovalent interactions between large molecules mediate many of life's functions in cells (Evans, 2001). The strength of interaction is the level of force to disrupt a bond on a particular time scale, knowing that these molecular interactions have limited lifetimes and thus fail under any level of force if pulled on for modest period of time (Evans, 1998). For instance, an isolated bond has no strength on times scales longer than its natural lifetime for spontaneous dissociation (entropy-driven): $t_{o f f}^{0}$. On the other hand, when a bond is pulled apart faster than needed for diffusive relaxation, it is classically thought to resist to detachment by a force given by the maximum gradient, $f_{\text {bond }} \sim(\partial E(x) / \partial x)_{\max }$, where $E(x)$ is the interaction potential or energy contour defined along the distance $(x)$ of separation. Thus, from the slow limit set by spontaneous transition, $t_{o f f}^{0}$, to the ultrafast limit for diffusive relaxation, $t_{D},\left(\approx 10^{-10}-10^{-9} \mathrm{~s}\right.$, i.e., the Brownian time), strength of non covalent bonds is governed by thermally activated kinetics under external force and depends on how force is applied over time. To describe bond kinetics, the simplest model of chemical 
binding is a single-sharp barrier model which takes into account the major impact of force on thermally activated kinetics that is an exponential amplification of the forward rate for dissociation characterized by a small force scale:

$k_{o f f}(f) \sim e^{-E_{b}(f) / k_{B} T}$

$E_{b}(f)=E_{b}^{0}-f \times x_{\beta}$

In this model, the energy barrier of a single bond is characterized in its simplest form by its height at zero force, $E_{b}^{0}$, and the distance in nanometer, or a fraction of it, at which this energy barrier is located, $x_{\beta}$. The impact of a force $f$ depends on how energy landscape is deformed by the force (given by Eq. 2). This model (Eq. 1 and 2) predicts that the rate of failure increases exponentially once force reaches a small scale $\left(k_{B} T / x_{\beta}\right)$, well below the adiabatic limit $-E_{b}(f) / k_{B} T$. Thermal activation sets the scale for force:

$f_{\beta}=\frac{k_{B} T}{x_{\beta}}$

$f_{\beta}$ provides a reference force scale that remains rather small since the thermal energy per molecule: $k_{B} T \approx 4 \mathrm{pN} \cdot \mathrm{nm}$, (with $k_{B}=1.381 \times 10^{-23} \mathrm{~J} / K$, Boltzman constant and $T$ absolute room temperature in ${ }^{\circ} K$ ), and $x_{\beta} \approx 0.1-1 \mathrm{~nm}$, (e.g., $f_{\beta} \approx 13 p N$ for integrin-VCAM linkage (Evans and Kinoshita, 2007); $f_{\beta} \approx 3-9 p N$ for adhesion between platelets and subendothelial matrix (Arya et al., 2005)). Using this scale, the rate of dissociation increases exponentially with force as proposed in the pioneering work by Bell (Bell, 1978):

$k_{o f f}(f)=k_{o f f}^{0} \times e^{f / f_{\beta}}$

Eq. 4 provides the dissociation rate of a single bond on which a force $f$ is exerted. It describes a stochastic phenomena analogue to the disintegration of radioactive substance. 
The dissociation rate $k_{o f f}(f)$ is linked to the probability $P(t)$ that the bond survives by a simple differential equation which provides the expression of $P(t)$ :

$\frac{d P(t)}{P(t)}=-k_{o f f}(f(t)) \times d t$ or equivalently $P(t)=\exp \left[-\int_{0}^{t} k_{o f f}\left(f\left(t^{\prime}\right)\right) \times d t^{\prime}\right]$

The prefactor of the dissociation rate given by the Bell model (Eq. 4) corresponds to the zero-force dissociation rate which classically depends on initial barrier height $E_{b}^{0}$.

$k_{o f f}^{0}=\frac{1}{t_{D}} e^{-E_{b}^{0} / k_{B} T}$

$k_{\text {off }}^{0}$ is the dissociation frequency for spontaneous escape in the absence of force already defined above $: k_{o f f}^{0}=\left(t_{o f f}^{0}\right)^{-1}$. Note that due to the exponential dependence shown by Eq. 6, bond lifetime is considerably higher than $t_{D}$. For instance, an integrin-VCAM bond with an energy barrier of around $20 k_{B} T$ (Evans and Kinoshita, 2007) has a bond lifetime $t_{o f f}^{0} \sim S$. Bond lifetimes would reach a few hours for energy barriers of $30 k_{B} T$. Due to the enormous gap in time scale between ultrafast Brownian diffusion and characteristic times of laboratory experiments $\left(\sim 10^{-4} \mathrm{~s}-\mathrm{min}\right)$, applied forces are essentially stationary on the Brownian scale.

\section{Multiple bonds}

Three assumptions are made to describe the dynamics of the failure of multiple uncorrelated bonds (Fig 1):

(i) The response to mechanical force of an assembly of bonds obeys to the principle issued from the single bond theory which states that the response to force depends on the deformation of energy landscape which thereby resembles a mountainous terrain made of multiple energy barriers (Evans, 2001; Tsukasaki et al., 2007), 
(ii) Associated bonds behave independently (non cooperative bonds). This supposes sufficient linkage for assembly but insufficient linkage to force the bonds to behave identically to reach simultaneous dissociation (cooperative bonds). Indeed, preserving the stochastic nature of a given bond assembly is probably the most pertinent assumption for the weak non covalent interactions that characterize interfacial biological bonds,

(iii) different bond configurations (e.g., parallel or zipper) result in different force regulations

(Fig 1). In parallel bonds, the load is redistributed among the surviving bonds (Evans, 2001). In "zipper" bond, only the leading bond experiences the entire loading force (Evans, 2001).

Whatever the predominant bond structure, multiple bonds can break at random times from first to last. Thus the global dissociation rate is a function of the instantaneous force and the distribution of rupture times can merely be described by a first order Markov process with time-dependent rate constants (Evans, 2001; Williams, 2003). Hence, far from thermal equilibrium, it is still possible to generalize the single-dynamic barrier model (Eq. 14) to more complex barriers. This model predicts that the transition rate for escape past a cascade of $N$ sharp barriers is governed by the sum of times $\left[k_{o f f}^{n \rightarrow n-1}(f)\right]^{-1}$ needed to transit individual barriers, starting from the bound state (Evans, 1998, 2001):

$K_{o f f}^{N \rightarrow 0}(f)=\frac{1}{\sum_{n=1}^{n=N}\left[k_{o f f}^{n \rightarrow n-1}(f)\right]^{-1}}$

According to this approach, each individual bond acts as the barrier of a more complex bond made of $N$ identical bonds. $k_{o f f}^{n \rightarrow n-1}(f)$ represents the transition rate through each barrier of a multivalent bond. It depends on bond configuration as shown below.

Equal sharing of the force between parallel bonds has the effect of multiplying the force scale for these bonds (Williams, 2003). Moreover, at each failure event, there are less and 
less possibilities of rebinding and therefore, the time scale for event is decreased and the dissociation rate is increased (Evans, 2001):

$k_{o f f}^{n \rightarrow n-1}=n k_{o f f}^{0} \times \exp \left(\frac{f}{n f_{\beta}}\right)$

For zipper bonds, the transition rate is constant at given force:

$k_{o f f}^{n \rightarrow n-1}=k_{o f f}^{0} \times \exp \left(\frac{f}{f_{\beta}}\right)$

Combining Eq. 6 and 7 leads to following expressions of the overall dissociation rates across $N$ bonds in parallel:

$K_{o f f}^{N \rightarrow 0}=k_{o f f}^{0}\left[\sum_{n=1}^{N} \frac{1}{n} \times \exp \left(-\frac{f}{n f_{\beta}}\right)\right]^{-1}$

And a pure analytical expression for zipper bonds:

$K_{o f f}^{N \rightarrow 0}=k_{o f f}^{0} \times\left(\frac{1}{N}\right) \times \exp \left(\frac{f}{f_{\beta}}\right)$

The series given by Eq. 10 has no analytical expression. However, we presently propose an approximation of this series:

$K_{o f f}^{N \rightarrow 0} \approx k_{o f f}^{0} \times \sqrt{2} \times\left(\frac{f}{N f_{\beta}}\right)^{0.5} \times \exp \left(\frac{f}{N f_{\beta}}\right)$

Eq. 11 and 12 show that the global dissociation rate (noted with capital letters: $K_{o f f}(f)$ ) for multiple bonds working uncooperatively can be written in the form of a generalized Bell's model:

$K_{o f f}(f)=K_{o f f}^{0}(f) \times \exp \left(\frac{f}{F_{\beta}}\right)$ 
The global dissociation rate, $K_{o f f}(f)$, its prefactor, $K_{o f f}^{0}(f)$, which may also depend on $f$, and the global force scale, $F_{\beta}$, show the kinetics of failure of multiple bonds with a necessarily composite energy barrier. Eq. 13 can be linked to an expression of probability $P(t)$ of bond survival having the form of Eq. 5. The new prefactor $K_{o f f}^{0}(f)$ characterizes how the width of the overall chemical energy barrier is modified by force (Evans and Ritchie, 1997). The exponential term is a damping factor which takes into account the higher likelihood of reaching the top of the new energy barrier lowered by force in proportion to the global thermal force scale, $F_{\beta}$.

For parallel bonds: $K_{o f f}^{0} \approx k_{o f f}^{0} \times \sqrt{2} \times\left(\frac{f}{F_{\beta}}\right)^{0.5}$ and $F_{\beta}=N f_{\beta}$

For zipper bonds: $K_{o f f}^{0}=k_{o f f}^{0} / N$ and $F_{\beta}=f_{\beta}$

Eq. 14 and 15 which relate collective bond properties to properties of single bond show explicitly how the collective bond organization modifies the kinetic adhesion parameters in the case of parallel and zipper configurations respectively.

In many experimental cases, the force experienced by an attachment is not constant but increases with time. Bonds under slow loading rates have long lifetimes but only withstand small forces, whereas bonds under fast loading have shorter lifetimes and withstand larger forces (Evans, 2001; Evans and Calderwood, 2007; Thomas et al., 2008). Hence, a classical way to explore the chemical energy landscape traversed in force-driven dissociation is to use adjustable force-ramp representing many loading rates from very slow to extremely fast. An alternative method has been recently proposed by Evans and Kinoshita (Evans and Kinoshita, 2007) in which a force-clamp method is applied to a single bond. The 
force-clamp method can be seen as the easiest method to establish the lifetime profile as a constant dissociation force is applied. Depending on the contours of energy function with the distance of separation (one or more chemical energy barriers), some bonds weaken as force increases (as predicted by Bell model, Eq. 4) while some other bonds strengthen as force increases (Sokurenko et al., 2008; Thomas, 2008; Thomas et al., 2008).

\section{Results and Discussion}

\section{Theoretical aspects}

Fig. 2 summarizes the theoretical predictions for lifetimes of multiple uncooperative bonds dissociating randomly (Eqs. 10-12). The lifetime of multiple bond ( $n=N)$ normalized by the bond lifetime of a single and isolated bond $(n=1)$ is plotted versus the number $N$ of identical bonds constituting the parallel and "zipper" configurations. The main message is that although the lifetime of the collective bonds still decreases with increasing force (as shown by Eq. 11 and 12), bonds organized collectively have a much higher capacity to stabilize the adhesive structure compared to isolated bonds. This stabilization effect increases with increasing the bond number for both parallel and zipper configurations. Note that by contrast, a series of uncorrelated bonds would dissociate faster than single bonds (Evans, 1998). In parallel and zipper bonds, this effect is particularly marked at the beginning of bond association: $n=2,3 \ldots$ Thus, the theory predicts that the early phase of lateral bond association is particularly efficient for stabilizing adhesion.

If both zipper and parallel configurations stabilize adhesion, the parallel configuration, where the dissociation force is equally divided between parallel bonds, 
reveals a marked effect of the force in the reduction of the global dissociation rate. This is basically due to the associated reduction in the exponential amplification classically described by Eqs 1 and 4 for single bonds, and generalized to collective bonds by Eqs. 11-13. However, at first glance, the force-dependent reduction in the dissociation rate of bonds characterized by a collective organization may be seen as a counter intuitive result if we forget to consider that both single and collective bonds still dissociate more under a force increase (Eqs. 4, 11 and 12). Indeed, the force-induced exponential decrease in bond lifetime is cut down by the collective bond organization, most remarkably in the parallel case. We underline here that the effect of increasing bond stabilization caused by a homogeneous force distribution has been under-considered before. This is nevertheless a fundamental mechanism for the control of cellular adhesion by force, including the force-dependence of dissociation or stabilization rates as well as the force-dependence of weakening or strengthening processes. In other words, lateral bond association (or clustering) is capable to promote an efficient reduction in the force-dependent increase in the dissociation rate of adhesion sites in a context where both collective and individual bonds all tend to fail at higher force.

By contrast, the zipper configuration provides a relevant example of the effect of a heterogeneous distribution of force on the global failure rate. For $N$ uncorrelated bonds in the zipper configuration, the global failure rate is divided by $N$, meaning that the stabilization mechanism directly depends on the bond number but, contrary to the parallel configuration, not on the force applied. It is equivalent to say that a heterogeneous force distribution limits the beneficial effect - in terms of stabilization - of dividing the force through several bonds. 
Another important message brought by the theoretical results plotted in Fig. 2 is that the analytical equation given by Eq. 10 provides a satisfactorily curve fitting while we noticed that previously proposed analytical forms such as in (Evans, 2001): $K_{o f f}(f) \approx k_{o f f}^{0} \times \frac{f}{N f_{\beta}} \times$ $\exp \left(\frac{f}{N f_{\beta}}\right)$, fail to provide a satisfactorily curve fitting. Note however that the form presently proposed (in Eq. 12) to fit the multiple bond lifetime (Eq. 10) remains consistent with the general form previously proposed to describe dissociation rates of bonds with necessarily complex energy barriers: $K_{o f f}(f) \approx k_{o f f}^{0} \times g(f) \times \exp \left(\frac{f}{N f_{\beta}}\right)$ with $k_{o f f}^{0} \times g(f)=K_{o f f}^{0}$ (Evans and Ritchie, 1997). Present results (Fig 2) bring a new confirmation of the initial intuitions of Evans (Evans, 2001; Evans and Ritchie, 1997) about the relevance of the Bell type model (Eq. 13) to describe dissociation rates of multiple parallel and zipper bonds which are individually in agreement with the Bell type model (Eq 11). Advantageously, these analytical expressions allow to delineate the specific effects of bond number and dissociation force on the kinetic adhesion parameters in various bond configurations (Fig 2).

\section{Comparison with experimental results}

\section{- Parallel bonds (bonds equally stretched by AFM tip)}

We attempted to analyze the feature of the parallel configuration described by the multiple bond theory above. The most straightforward example is provided by the reference data obtained by the group of A. Noy (Friddle et al., 2007; Sulchek et al., 2005) who used dynamic force spectroscopy via AFM to characterize binding of several individual singlechain variable fragments (scFv) antibody to the Mucin1 (MUC1) peptide (MUC1-Ab). This 
interaction is the main targeting mechanism for a family of experimental radioimmunotherapics for cancer treatment (Albrecht et al., 2004). Indeed, clinical researchers have used multivalency of protein adhesion as an affinity-enhancing approach in a variety of immunotherapies and imaging techniques to target specific tissues (Souriau and Hudson, 2003). Noteworthy, authors (Friddle et al., 2007; Sulchek et al., 2005) found a direct experimental verification of the uncorrelated multiple bond rupture model because they were able to impose a uniform force distribution through a few parallel bonds and compared with the single bond. A remarkable confirmation of the theoretical predictions presented above was brought by these authors because the dissociation rate found for two (respectively three) bonds in parallel drop precipitously compared to the single (respectively two) bond. The present theoretical approach revealed that in addition to the bond number effect, the effect of the force magnitude above the reference force scale, was also a critical parameter. Indeed, in these AFM experiments, force increased sharply in parallel to the bond number (e.g., $f / f_{\beta} \approx 10$ for $N=1, f / f_{\beta} \approx 20$ for $N=2, f / f_{\beta} \approx 30$ for $N=3$ ). Theoretical results shown in Fig. 2 reveal that as force increases, the bond number effect on dissociation rate is dramatically increased in uncorrelated parallel bonds with homogeneous force distribution. Present data demonstrate that the main beneficial effect of multivalent interactions compared to single bond is the reduction on the kinetic off-rate and the corresponding increase in lifetime to the extent that the force distribution remains homogeneous. Such a parallel configuration would certainly be desired to improve the binding efficiency of receptor-targeted immunotherapic treatment (Souriau and Hudson, 2003).

\section{- Zipper bonds (Bonds unequally stretched by shear flow)}


The viscous shear flow allows studying the motion of receptor-coated cells or particles along a flat ligand-coated surface. At low hydrodynamic force (e.g., a few pN) and for low ligand concentrations (e.g., from 9 to 170 ligands $/ \mu \mathrm{m}^{2}$ in (Smith et al., 1999)), detectable cell/particle arrests reveal single molecular bond formation (Kaplanski et al., 1993; Pierres et al., 1996a; Smith et al., 1999). Neglecting rebinding if receptor density is low, the dissociation rate could be deduced from the slope of the natural log of number of cell-surface interactions versus the duration of each event (Kaplanski et al., 1993; Pierres et al., 1996b; Smith et al., 1999; Tissot et al., 1992). In (Smith et al., 1999), the measured unstressed dissociation rates $k_{\text {off }}^{0}$ of single bond remained in a close range of values: $2.4-3.8$ $\mathrm{s}^{-1}$, in spite of the wide variety of ligand tested (i.e., P-selectin, E-selectin, L-selectin and PNAd). This is consistent with data obtained by other authors on the same cells (Alon et al., 1998; Alon et al., 1995; Kaplanski et al., 1993; Smith et al., 1999). The shear stressdependence on dissociation rates, $k_{\text {off }}(f)$, was affected by the change in receptor-ligand affinity to take values in a higher range: $5-36 \mathrm{~s}^{-1}$ as expected from Bell model (Eq. 4) (Smith et al., 1999). Beyond, this type of experiment is important as it allows to study the flowenhanced cell adhesion mechanisms (Zhu et al., 2008). Concerning the limits of the single bond assumption in sheared cells, it has been suspected that, as ligand density or shear stress increases, an increased number of multiple bond events is involved in cell adhesion. However, their effect was not thought to deeply affect the estimated values of dissociation rate $k_{\text {off }}(f)$ (Smith et al., 1999).

In single bond models of cell arrest under shear, an oblique direction of the bond force reacting to the shear force is assumed (Chang and Hammer, 1999; Smith et al., 1999; Zhu et al., 2008). Hydrodynamic theory predicts that once a bond is created with the 
substrate, it tends to re-equilibrate the translational and rotational velocity components at the cell wall, thus cancelling out the slip velocity (Chang and Hammer, 1999; Hammer and Apte, 1992). This suggests that the direction of bond traction during shear flow is necessarily oblique, i.e., resulting from tangential and normal tensile (or compressive) force components at an adhesive interface (Gallant et al., 2005). Based on previous analysis (Evans, 1985; Ward and Hammer, 1993), detachment of a sheared cell is expected to occur via peeling of the leading edge of the cell. Accordingly, bond loading is highly non uniform along the contact area since bond forces are maximal at the periphery and decay rapidly toward the center of the cell (Zhu et al., 2008). Thus, in shear flow experiments where independent bonds start to respond collectively, i.e., at increasing shear (Zhu et al., 2008) or at increasing ligand/receptor concentrations (Gallant et al., 2005), the zipper configuration appears to be a satisfactorily model because force distribution on bonds is highly non uniform. It means that the huge effects on lifetimes of multiple parallel bonds (Fig 2) such as those reported from AFM experiments (Sulchek et al., 2006) could not be expected in shear flow experiments. Accordingly, studying cell adhesion strengthening in sheared fibroblasts, Gallant et al. (Gallant et al., 2005) were surprised to observe that parallel association of integrins by focal adhesion (respectively clustering) accounts for only $30 \%$ (respectively 60\%) of the strengthening response to shear. We presently postulate that this result is linked to the highly unequal distribution of the resultant force during shear. These mechanical factors would clearly favor the zipper configuration which exhibits the lowest sensitivity to the bond number and no force-dependence for the prefactor of the dissociation rate. We conclude that the homogeneous or heterogeneous character of the force distribution, which is indeed associated to the resulting direction of the force applied on interfacial bonds, is a determinant factor of the kinetics of adhesion. 


\section{Significance of present results}

Theoretical approach and experimental results consistently show that the distribution of force amongst bonds organized collectively and working stochastically fundamentally affects the kinetics of adhesion. Depending on the size of adhesion site, the scale at which these mechanisms operate cover a wide range of scales: from the nanometer scale for the weak initial adhesion sites constituted by only a few molecules (e.g., integrins and talins) to ten micrometers for the mature focal adhesion which contains more than fifty adhesion proteins (Zamir and Geiger, 2001). The list of adhesion molecules continue to grow and key mechanisms that regulate signal transduction pathways and modulate adhesion assembly still need to be identified (Lo, 2006). Future challenges include the determination of how these molecules work together as a physico-chemical complex and how their coupling contributes to an adapted biological response. This reinforces the need of calculating stress/strain fields exerted on living tissues at various scales and certainly up to the smallest molecular scales, e.g., in the lung (Suki and Bates, 2008). In this perspective, computational fluid/solid mechanics appears for the future as an indispensible tool to get mechanical parameters at various scales, in various living structures and functional conditions, e.g., in the lung (Elad et al., 2008). More particularly, we find that, if the force exerted on a given adhesion site is distributed homogeneously amongst the bonds, one may expect a force-dependent stabilization process which dramatically depends on bond number. Noteworthy, the stochastic theory of parallel bonds stretched uniformly predicts a drastic reduction in the dissociation rate compared to single bonds. Unexpectedly, the 
relative decay in dissociation rate, (i.e., increase in lifetime), of collective bonds compared to single bond is enhanced by increasing stretching forces.

In the absence of precise knowledge about the force distribution in living tissues down to the nanoscale, it is easier to consider that the heterogeneous force distribution better fits the standard biological conditions. However, this is not necessarily true. We can say that the parallel organization of bonds constitutes an impressive means to cut down the straightforward process of force-induced reduction in lifetime. This remarkable property of parallel bonds - and to a lesser extent of zipper bonds -has not been so clearly pointed out before. Experiments by AFM constitute the most ideal conditions in which multiple parallel bonds can be stretched uniformly and indeed these AFM results seem consistent with the theory (Sulchek et al., 2005). As soon as force distribution on multiple bonds is heterogeneous, the stabilization process will still be effective but much less marked than in the case of uniform distribution. This is because the totality of the force is exerted on the leading bond(s), i.e., the proper of the zipper configuration, and thereby the rupture force of the leading bond, is rapidly attained. We point out here that the force distribution applied on adhesion sites of sheared cells is likely highly heterogeneous. This situation most likely corresponds to many biological situations including the frequent case of cells sheared by the flowing blood and also the pathological cases in general. Taking again the example of the deep lung, the highly elevated stress sites found in diseased emphysematous lungs suggests that heterogeneous stress distribution could weaken adhesion sites in the deep lung and thereby contribute to the disease (Gefen et al., 1999). Finally, another consequence of the present approach is to show that, as soon as bonds are multiple, with the same bond number, but stretched by different force/stress fields, one can expect very different data in terms of bond kinetics. Indeed, specific bond kinetics experiments performed with tightly 
controlled homogeneous and heterogeneous force distributions have to be designed to further test the presently developed concept.

\section{Acknowledgement}

For this work, we acknowledge receipt of a grant from Agence Nationale de la Recherche (ANR-09-PIRI-002-03).

\section{References}

Albrecht, H., Burke, P.A., Natarajan, A., Xiong, C.Y., Kalicinsky, M., DeNardo, G.L., DeNardo, S.J., 2004. Production of soluble ScFvs with C-terminal-free thiol for site-specific conjugation or stable dimeric ScFvs on demand. Bioconjug Chem 15, 16-26.

Alon, R., Chen, S., Fuhlbrigge, R., Puri, K.D., Springer, T.A., 1998. The kinetics and shear threshold of transient and rolling interactions of L-selectin with its ligand on leukocytes. Proc Natl Acad Sci U S A 95, 11631-11636.

Alon, R., Hammer, D.A., Springer, T.A., 1995. Lifetime of the P-selectin-carbohydrate bond and its response to tensile force in hydrodynamic flow. Nature $374,539-542$.

Andre, P., Benoliel, A.M., Capo, C., Foa, C., Buferne, M., Boyer, C., Schmitt-Verhulst, A.M., Bongrand, P., 1990. Use of conjugates made between a cytolytic T cell clone and target cells to study the redistribution of membrane molecules in cell contact areas. J Cell Sci 97 ( Pt 2), 335-347. 
Arya, M., Kolomeisky, A.B., Romo, G.M., Cruz, M.A., Lopez, J.A., Anvari, B., 2005. Dynamic force spectroscopy of glycoprotein Ib-IX and von Willebrand factor. Biophys J 88, 43914401.

Ashkin, A., 1992. Forces of a single-beam gradient laser trap on a dielectric sphere in the ray optics regime. Biophys J 61, 569-582.

Ashkin, A., Schutze, K., Dziedzic, J.M., Euteneuer, U., Schliwa, M., 1990. Force generation of organelle transport measured in vivo by an infrared laser trap. Nature 348, 346-348.

Balaban, N.Q., Schwarz, U.S., Riveline, D., Goichberg, P., Tzur, G., Sabanay, I., Mahalu, D., Safran, S., Bershadsky, A., Addadi, L., Geiger, B., 2001. Force and focal adhesion assembly: a close relationship studied using elastic micropatterned substrates. Nat Cell Biol 3, 466-472.

Bell, G.I., 1978. Models for the specific adhesion of cells to cells. Science 200, 618-627.

Bell, G.I., Dembo, M., Bongrand, P., 1984. Cell adhesion. Competition between nonspecific repulsion and specific bonding. Biophys J 45, 1051-1064.

Binnig, G., Quate, C.F., Gerber, C., 1986. Atomic force microscope. Phys Rev Lett 56, 930-933. Bongrand, P., Golstein, P., 1983. Reproducible dissociation of cellular aggregates with a wide range of calibrated shear forces: application to cytolytic lymphocyte target cell conjugates. J Immunol Methods 58, 209-224.

Bruinsma, R.F., 2005. Theory of Force Regulation by Nascent Adhesion Sites. Biophys J.

Capo, C., Garrouste, F., Benoliel, A.M., Bongrand, P., Depieds, R., 1981. Non-specific binding by macrophages: evaluation of the influence of medium-range electrostatic repulsion and short-range hydrophobic interaction. Immunol Commun 10, 35-43.

Chang, K.C., Hammer, D.A., 1999. The forward rate of binding of surface-tethered reactants: effect of relative motion between two surfaces. Biophys J 76, 1280-1292. 
Choquet, D., Felsenfeld, D.P., Sheetz, M.P., 1997. Extracellular matrix rigidity causes strengthening of integrin-cytoskeleton linkages. Cell 88, 39-48.

Cluzel, C., Saltel, F., Lussi, J., Paulhe, F., Imhof, B.A., Wehrle-Haller, B., 2005. The mechanisms and dynamics of (alpha)v(beta)3 integrin clustering in living cells. J Cell Biol 171, 383392.

Discher, D.E., Janmey, P., Wang, Y.L., 2005. Tissue cells feel and respond to the stiffness of their substrate. Science 310, 1139-1143.

Elad, D., Wolf, M., Keck, T., 2008. Air-conditioning in the human nasal cavity. Respir Physiol Neurobiol 163, 121-127.

Evans, E., 1998. Energy landscapes of biomolecular adhesion and receptor anchoring at interfaces explored with dynamic force spectroscopy. Faraday Discuss, 1-16.

Evans, E., 2001. Probing the relation between force--lifetime--and chemistry in single molecular bonds. Annu Rev Biophys Biomol Struct 30, 105-128.

Evans, E., Kinoshita, K., 2007. Using force to probe single-molecule receptor-cytoskeletal anchoring beneath the surface of a living cell. Methods Cell Biol 83, 373-396.

Evans, E., Ritchie, K., 1994. Probing molecular attachments to cell surface receptors: image of stochastic bonding and rupture processes, in: J. Rabe, H. E. Gaub, Hansma, P.K. (Eds.), Scanning Probe Microscopies and Molecular Materials. Kluwer Publishing, Amsterdam.

Evans, E., Ritchie, K., 1997. Dynamic strength of molecular adhesion bonds. Biophys J 72, 1541-1555.

Evans, E., Ritchie, K., Merkel, R., 1995. Sensitive force technique to probe molecular adhesion and structural linkages at biological interfaces. Biophys J 68, 2580-2587.

Evans, E.A., 1985. Detailed mechanics of membrane-membrane adhesion and separation. II. Discrete kinetically trapped molecular cross-bridges. Biophys J 48, 185-192. 
Evans, E.A., Calderwood, D.A., 2007. Forces and bond dynamics in cell adhesion. Science 316, 1148-1153.

Friddle, R.W., Sulchek, T.A., Albrecht, H., De Nardo, S.J., Noy, A., 2007. Counting and breaking individual biological bonds: Force spectroscopy of tethered ligand-receptor pairs. Current Nanoscience 3, 41-48.

Galbraith, C.G., Yamada, K.M., Sheetz, M.P., 2002. The relationship between force and focal complex development. J Cell Biol 159, 695-705.

Gallant, N.D., Michael, K.E., Garcia, A.J., 2005. Cell adhesion strengthening: contributions of adhesive area, integrin binding, and focal adhesion assembly. Mol Biol Cell 16, 43294340.

Gefen, A., Elad, D., Shiner, R.J., 1999. Analysis of stress distribution in the alveolar septa of normal and simulated emphysematic lungs. J Biomech 32, 891-897.

Geiger, B., Bershadsky, A., 2001. Assembly and mechanosensory function of focal contacts. Curr Opin Cell Biol 13, 584-592.

Giannone, G., Jiang, G., Sutton, D.H., Critchley, D.R., Sheetz, M.P., 2003. Talin1 is critical for force-dependent reinforcement of initial integrin-cytoskeleton bonds but not tyrosine kinase activation. J Cell Biol 163, 409-419.

Hammer, D.A., Apte, S.M., 1992. Simulation of cell rolling and adhesion on surfaces in shear flow: general results and analysis of selectin-mediated neutrophil adhesion. Biophys J $63,35-57$.

Kaplanski, G., Farnarier, C., Tissot, O., Pierres, A., Benoliel, A.M., Alessi, M.C., Kaplanski, S., Bongrand, P., 1993. Granulocyte-endothelium initial adhesion. Analysis of transient binding events mediated by E-selectin in a laminar shear flow. Biophys J 64, 1922-1933. 
Leckband, D.E., Israelachvili, J.N., Schmitt, F.J., Knoll, W., 1992. Long-range attraction and molecular rearrangements in receptor-ligand interactions. Science 255, 1419-1421.

Lo, S.H., 2006. Focal adhesions: what's new inside. Dev Biol 294, 280-291.

Pasternak, C., Elson, E.L., 1985. Lymphocyte mechanical response triggered by cross-linking surface receptors. J Cell Biol 100, 860-872.

Pierres, A., Benoliel, A.M., Bongrand, P., 1996a. Measuring bonds between surfaceassociated molecules. J Immunol Methods 196, 105-120.

Pierres, A., Benoliel, A.M., Bongrand, P., van der Merwe, P.A., 1996b. Determination of the lifetime and force dependence of interactions of single bonds between surface-attached CD2 and CD48 adhesion molecules. Proc Natl Acad Sci U S A 93, 15114-15118.

Simson, D.A., Ziemann, F., Strigl, M., Merkel, R., 1998. Micropipet-based pico force transducer: in depth analysis and experimental verification. Biophys J 74, 2080-2088.

Smith, M.J., Berg, E.L., Lawrence, M.B., 1999. A direct comparison of selectin-mediated transient, adhesive events using high temporal resolution. Biophys J 77, 3371-3383.

Sokurenko, E.V., Vogel, V., Thomas, W.E., 2008. Catch-bond mechanism of force-enhanced adhesion: counterintuitive, elusive, but ... widespread? Cell Host Microbe 4, 314-323.

Souriau, C., Hudson, P.J., 2003. Recombinant antibodies for cancer diagnosis and therapy. Expert Opin Biol Ther 3, 305-318.

Suki, B., Bates, J.H., 2008. Extracellular matrix mechanics in lung parenchymal diseases. Respir Physiol Neurobiol 163, 33-43.

Sulchek, T., Friddle, R.W., Noy, A., 2006. Strength of multiple parallel biological bonds. Biophys J 90, 4686-4691. 
Sulchek, T.A., Friddle, R.W., Langry, K., Lau, E.Y., Albrecht, H., Ratto, T.V., DeNardo, S.J., Colvin, M.E., Noy, A., 2005. Dynamic force spectroscopy of parallel individual Mucin1antibody bonds. Proc Natl Acad Sci U S A 102, 16638-16643.

Thomas, W., 2008. Catch bonds in adhesion. Annu Rev Biomed Eng 10, 39-57.

Thomas, W.E., Vogel, V., Sokurenko, E., 2008. Biophysics of catch bonds. Annu Rev Biophys $37,399-416$.

Tissot, O., Pierres, A., Foa, C., Delaage, M., Bongrand, P., 1992. Motion of cells sedimenting on a solid surface in a laminar shear flow. Biophys J 61, 204-215.

Tsukasaki, Y., Kitamura, K., Shimizu, K., Iwane, A.H., Takai, Y., Yanagida, T., 2007. Role of multiple bonds between the single cell adhesion molecules, nectin and cadherin, revealed by high sensitive force measurements. J Mol Biol 367, 996-1006.

Vogel, V., Sheetz, M.P., 2009. Cell fate regulation by coupling mechanical cycles to biochemical signaling pathways. Curr Opin Cell Biol 21, 38-46.

Ward, M.D., Hammer, D.A., 1993. A theoretical analysis for the effect of focal contact formation on cell-substrate attachment strength. Biophys J 64, 936-959.

Williams, P.M., 2003. Analytical descriptions of dynamic force spectroscopy: behaviour of multiple connections. Analytica Chimica Acta 479, 107-115.

Zamir, E., Geiger, B., 2001. Components of cell-matrix adhesions. J Cell Sci 114, 3577-3579.

Zhu, C., Yago, T., Lou, J., Zarnitsyna, V.I., McEver, R.P., 2008. Mechanisms for flow-enhanced cell adhesion. Ann Biomed Eng 36, 604-621. 


\section{Figure Legend}

Figure 1: The two main configurations considered in the present study of the kinetics of multiple bonds are the parallel configuration (upper schema) and the zipper configuration (lower schema). In the parallel configuration, the dissociation force, $f$, is uniformly distributed amongst identical bonds. After breaking, force is redistributed amongst the remaining bonds. In the zipper configuration, the leading bond experiences the entire force until breaking while it is passed on to the next. In the two cases, it is assumed that bonds break at random times from first to last, (i.e., described by a Makovian sequence) because the lack of mechanical linkage between bonds ensures a sufficient independence between bonds.

Figure 2: The kinetics of uncorrelated multiple independent bonds is compared to the kinetics of an individual bond. The quantity $\frac{\left[K_{o f f}(f)\right]^{-1}}{\left[k_{o f f}(f)\right]^{-1}}$ represents the ratio between two lifetimes: the multiple bonds to single bond lifetimes at a given force $f$. The lifetime ratio of multiple to single bonds is plotted on a logarithmic scale as a function of the bond number $N$ for different values of the separation force given by the ratio $\frac{f}{f_{\beta}}$ (presently in the range: 230). Lifetime being by definition the inverse of the dissociation rate, this ratio provides equivalently the evolution of the kinetic rate constant, (or dissociation rate), of the single bond comparatively to the collective bond on which the same force is exerted. In parallel bonds, the force amplifies - by orders of magnitude - the $\mathrm{N}$-dependent increase in relative lifetime. In zipper bonds, the ratio of multiple to single bond lifetimes also increases, but remains proportional to $N$. The zipper-bond relationship can be superimposed to the 
parallel-bond relationship obtained for $\frac{f}{f_{\beta}}=2$. Diamond symbols represent the relationships predicted by the stochastic theory of uncorrelated multiple parallel bonds (see Eq. 10 and Williams (Williams, 2003)). Empty squares represent the relationships predicted by the theory of uncorrelated multiple zipper bonds (see Eq. 11 and Williams (Williams, 2003) and Evans (Evans, 2001)). The continuous curves correspond to the proposed adjustment of the stochastic theory using the explicit form given in Eq. 12 to describe the kinetics of uncorrelated parallel bonds. 
Figure 1
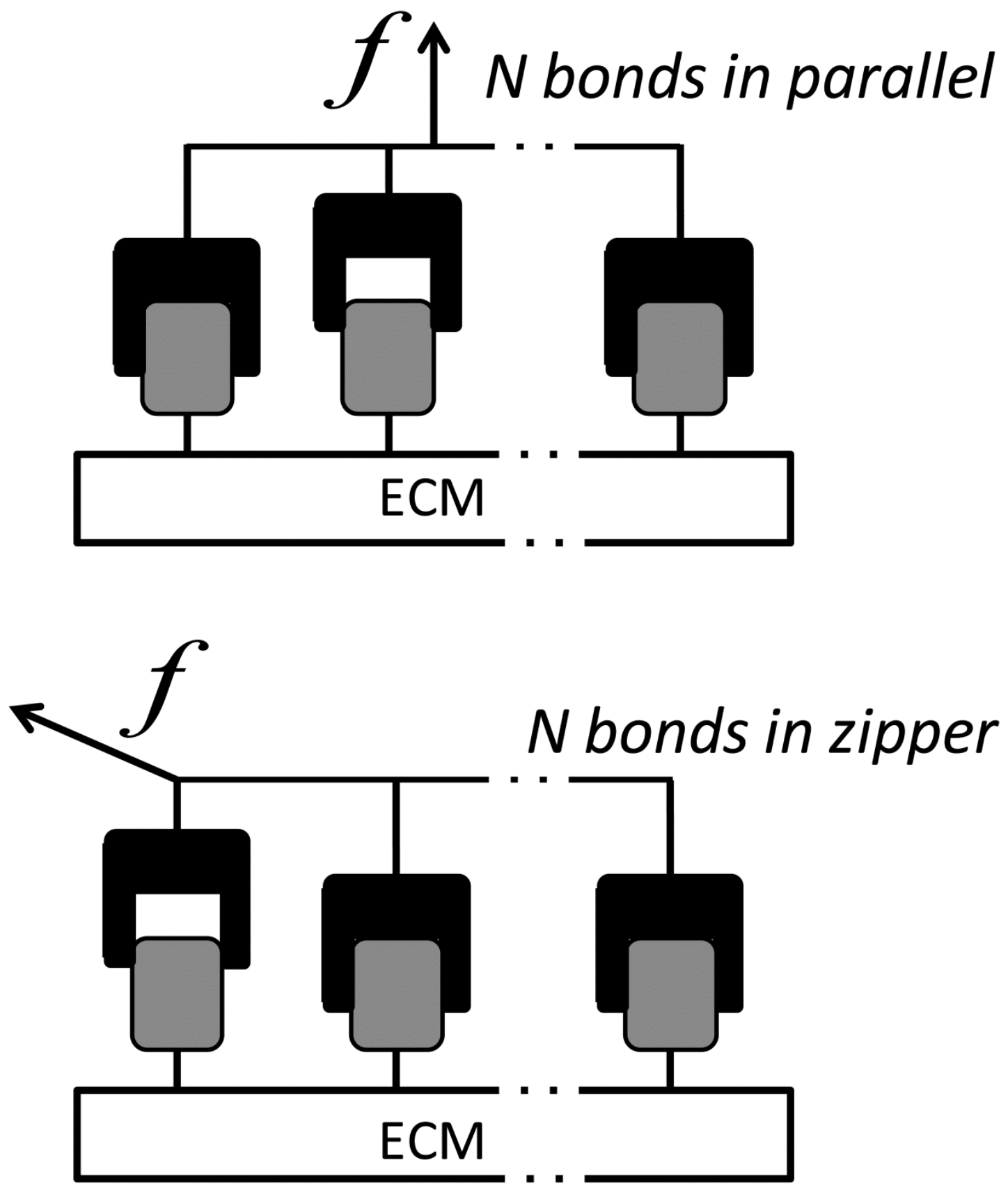
Figure 2

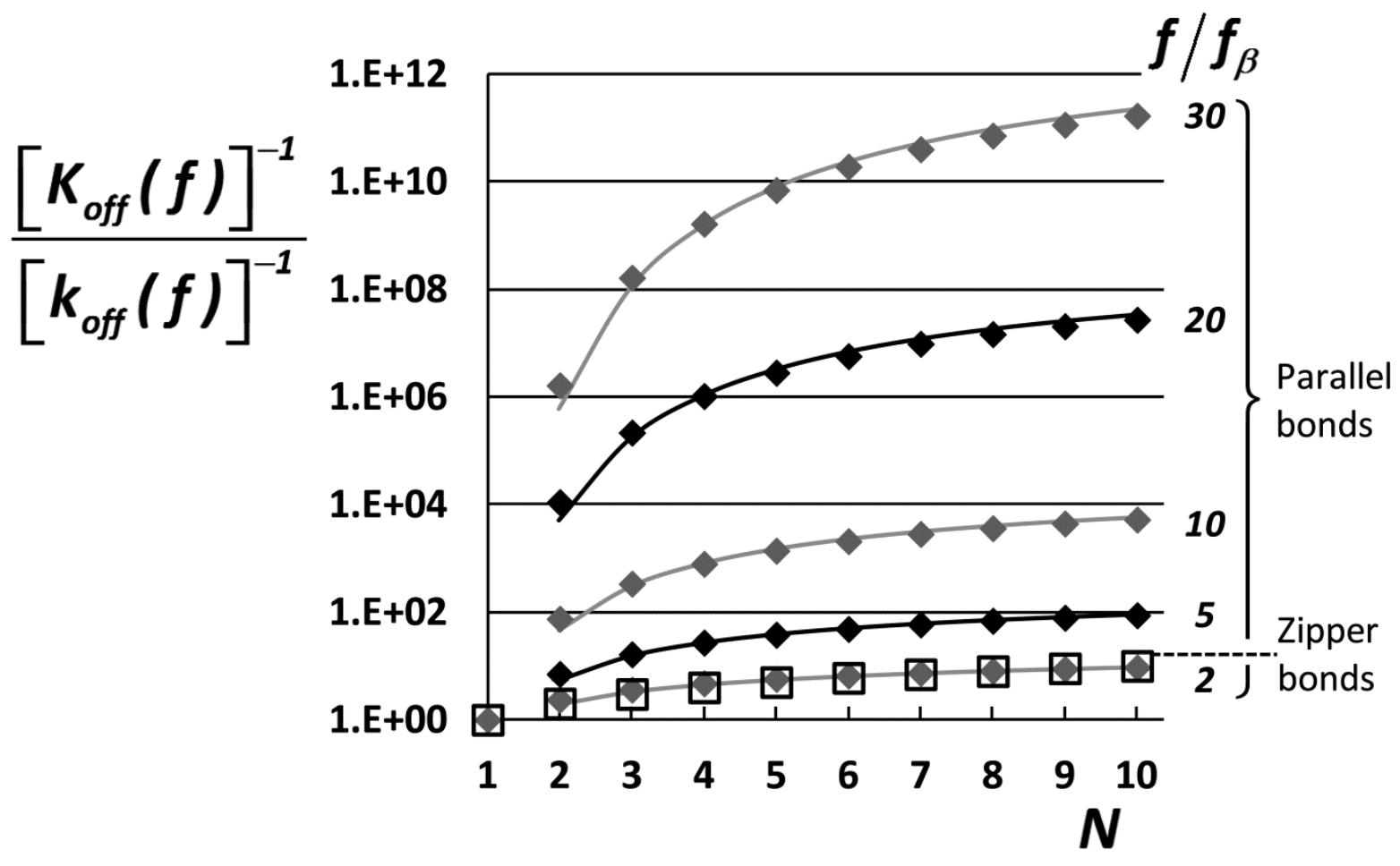

